\title{
On the spinning axis representation
}

\author{
J. A. de Azcárraga \\ Física Matemática and GIFT, Facultad de Ciencias, Salamanca, Spain \\ L. Navarro \\ Departamento de Física Teórica and GIFT, Facaltad de Ciencias, Barcelona, Spain \\ (Received 10 April 1974) \\ A new spinning axis representation is introduced. It allows us to calculate the evolution operator of a \\ system with slowly varying time-dependent Hamiltonian with the desired degree of approximation in \\ the parameter used for describing its dynamical evolution. The procedure is compared with a \\ previously existing one and applied to a simple example.
}

\section{INTRODUCTION}

The application of perturbative methods to systems described by slowly varying time-dependent Hamiltonians allows one to obtain asymptotic rather than convergent expansions for the solution of the Schrödinger equation. This is usually done making use of the "spinning axis representation" (SAR) which is defined by the fact that, in it, the Hamiltonian eigenvectors are independent of time. The application of the SAR to the Schrödinger equation satisfied by the evolution operator leads to an equation which makes easy the use of timedependent perturbation theory. In this way the ordinary adiabatic theorem is obtained, which can be established in the following form: "If the state of a system is represented at $t=t_{0}$ by the ket $\left|\epsilon_{j}\left(t_{0}\right)\right\rangle$ which is a solution of the eigenvalue equation $H(t)\left|\epsilon_{j}(t)\right\rangle=\epsilon_{j}(t)\left|\epsilon_{j}(t)\right\rangle$ for $t=t_{0}$, at $t=t_{1}$ the state of the system is represented by the ket $\left|\epsilon_{f}\left(t_{1}\right)\right\rangle$ which is solution of the same equation for $t=t_{1}$." This result is more accurate the stronger is the inequality

$$
|\max (\alpha) / \min (\omega)|^{2} \ll 1,
$$

where $\max (\alpha)$ is the maximum value of the "angular velocity" of the eigenaxes of the Hamiltonian, and $\min (\omega)$ is the minimum value of the Bohr frequency for transitions between eigenstates. ${ }^{1}$

In the usual treatment of the adiabatic theorem it is supposed that the eigenvalues of the Hamiltonian remain separated during the transition period $T=t_{1}-t_{0}$. However, the adiabatic theorem remains valid if this assumption is violated ${ }^{2}$; in the same way, the hypothesis of discrete spectrum is not necessary. ${ }^{3}$ These restrictions are introduced for the sake of simplicity, and we shall maintain them here.

The ordinary adiabatic theorem, valid in the first approximation of order $1 / T$, may be generalized to any order without requiring special properties for the Hamiltonian. To this aim, instead of applying perturbation theory to the Schrödinger equation in the SAR as is done in the usual adiabatic theorem, a new change of representation is made ${ }^{4}$ which fixes the eigenstates of the operator which plays the role of the Hamiltonian in the SAR of the Schrodinger equation. This transformation defines a new SAR, and the procedure can be repeated to get the desired order of approximation. In this way, to obtain the evolution operator $U$ of a system up to terms of order $(1 / T)^{n}$, n succesive transformations ( $n$ SAR) are required. This makes the method rather cumbersome in practice, since to obtain the form of the $n$th transformation it is necessary to know the explicit form of the previous $(n-1)$ SAR.

In this paper we propose a method which allows one to obtain the evolution operator $U$ to the desired degree of approximation by means of only one change of representation which defines a generalized SAR (GSAR). The action of the operator defining the GSAR is obviously equivalent to the combined action of the $n$ operators $R^{i}$ defining the succesive SAR of Ref. 4.

In order to show this, we describe briefly in Sec. 2 the ordinary SAR and introduce in Sec. 3 the GSAR. Finally, in Sec. 4 we consider a simple example which illustrates the method.

\section{THE ORDINARY SPINNING AXIS REPRESENTATION}

Let us consider a quantum system whose dynamical evolution is determined by its Hamiltonian $H(t)$. We shall assume a good behavior for it and its derivatives in the interval $t_{0} \leqslant t \leqslant t_{1}$ in which we consider the variation of $H(t)$. We shall measure the time by means of the variable $\tau=t-t_{0} / T$, where $T=t_{1}-t_{0}$. We shall suppose $T$ large (adiabatic evolution) in the sense that the inequality (1.1) holds.

Let $U(\tau)$ be the evolution operator of the system described by $H(\tau) . U(\tau)$ is then the solution of

$$
i \hbar \frac{d}{d \tau} U(\tau)=T H(\tau) U(\tau)
$$

with the initial condition

$$
U(0)=I \text {. }
$$

After applying the unitary transformation $A(\tau),(2.1)$ takes the form

$$
i \hbar \frac{d}{d \tau} U^{(A)}(\tau)=T\left[H^{(A)}(\tau)-(1 / T) K^{(A)}(\tau)\right] U^{(A)}(\tau)
$$

where $U^{(A)}(\tau) \equiv A^{\dagger}(\tau) U(\tau)$ is the new evolution operator, $H^{(A)}(\tau) \equiv A^{\dagger}(\tau) H(\tau) A(\tau)$ is the transformed Hamiltonian, and $K^{(A)}(\tau) \equiv A^{\dagger}(\tau) K(\tau) A(\tau)$, with $K(\tau)$ being the generator of the transformation $A(\tau)$, i. e. , the solution of the differential equation

$$
i \hbar \frac{d}{d \tau} A(\tau)=K(\tau) A(\tau)
$$

subjected to the initial condition $K(0)=0$. Note that in (2.3) the operator which plays the role of the Hamilton- 
ian of the new representation differs from the transformed Hamiltonian in the small term $-(1 / T) K^{(A)}(\tau)$.

It is clear that the equation satisfied by the evolution operator is easily integrated if the projectors of the eigenvectors of the Hamiltonian do not depend on time. Thus, to calculate $U$ in the adiabatic approximation, $A$ (SAR) is defined in such a way that $A\left|\epsilon_{j}(0)\right\rangle=\left|\epsilon_{j}(\tau)\right\rangle$, $j=1,2, \cdots$, and then the transformed Hamiltonian $H^{(A)}=\sum_{j} \epsilon_{j}(\tau)\left|\epsilon_{j}(0)\right\rangle\left\langle\epsilon_{j}(0)\right|$ is treated by perturbation theory. The procedure can be improved by finding a new SAR for the operator $\left[H^{(A)}(\tau)-(1 / T) K^{(A)}(\tau)\right]$ of $(2.3)$. In this way one is naturally led to the method of Ref. 4 in which, to get an approximation of $n$th order, $n$ succesive SAR are needed. Instead of doing this, however, we look in the next section for a unique transformation which will allow us to calculate $U$ with the same degree of approximation.

\section{THE NEW TRANSFORMATION}

Let us write (2.3) in the form

$$
\begin{aligned}
i \hbar \frac{d}{d \tau} U^{(s)}(\tau) & =T S^{\dagger}(\tau)\left(H(\tau)-\frac{1}{T} K(\tau)\right) S(\tau) U^{(s)}(\tau) \\
& \equiv T S^{\dagger}(\tau) H^{\prime}(\tau) S(\tau) U^{(s)}(\tau) \\
& =T H^{\prime(s)}(\tau) U^{(s)}(\tau) .
\end{aligned}
$$

If it were possible to find a transformation $S$ such that $H^{\prime(s)}(\tau)\left[\right.$ not $\left.H^{(s)}(\tau)\right]$ had time-independent eigenvectors, i. e., such that

$$
S^{\dagger}(\tau)\left|\epsilon_{j}(\tau)\right\rangle=\left|\epsilon_{j}(0)\right\rangle, \quad j=1,2, \cdots,
$$

where

$$
H^{\prime}(\tau)\left|\epsilon_{j}(\tau)\right\rangle=\epsilon_{j}(\tau)\left|\epsilon_{j}(\tau)\right\rangle
$$

(3.1) could be exactly solved giving for $U$,

$$
U(\tau)=S(\tau) \sum_{j} \exp \left[-i T \varphi_{j}(\tau) / \hbar\right]\left|\epsilon_{j}(0)\right\rangle\left\langle\epsilon_{j}(0)\right|
$$

with

$$
\varphi_{j}(\tau)=\int_{0}^{\tau} \epsilon_{j}\left(\tau^{\prime}\right) d \tau^{\prime} .
$$

It is obvious that $S$, which defines the GSAR, cannot be found exactly: A simple inspection of $(3,1)-(3.3)$ shows that, to find $S(\tau)$, one needs to solve the eigenvalue problem for $H^{\prime}(\tau)$ and that, with this aim, it is necessary to know the generator $K$ of the transformation $S$ which enters in the definition of $H^{\prime}(\tau)$. We show next, however, that it is possible to determine $S$ (and thus $U$ ) with the desired degree of approximation taking into account that $T$ is large in the already specified sense.

\section{A. First approximation}

Let $\left|\epsilon_{j}^{(1)}(\tau)\right\rangle, S^{(1)}(\tau), K^{(1)}(\tau)$, and $\epsilon_{j}^{(1)}(\tau)$ be the results of this approximation (the order corresponds to that in which the practical calculation is to be made). Since

$$
H^{\prime}(\tau)=H(\tau)-(1 / T) K(\tau)
$$

in the first approximation, we find that $\left|\epsilon_{j}^{(1)}(\tau)\right\rangle$ are defined through

$$
H(\tau)\left|\epsilon_{j}^{(1)}(\tau)\right\rangle=\epsilon_{j}^{(1)}(\tau)\left|\epsilon_{j}^{(1)}(\tau)\right\rangle, \quad j=1,2, \cdots,
$$

and

$$
S^{(1)}(\tau)\left|\epsilon_{j}^{(1)}(0)\right\rangle=\left|\epsilon_{j}^{(1)}(\tau)\right\rangle .
$$

$S^{(1)}(\tau)$ clearly corresponds to the transformation $A(\tau)$ mentioned in Sec. 2 and to $R^{(1)}(\tau)$ of Ref. 4. The generator $K^{(1)}(\tau)$ can also be calculated, and is given by

$$
K^{(1)}(\tau)=i \hbar \sum_{j}\left(\frac{d}{d \tau}\left|\epsilon_{j}^{(1)}(\tau)\right\rangle\right)\left\langle\epsilon_{j}^{(1)}(\tau)\right| .
$$

The choice in (3.9) implies that the arbitrariness of the phase of $\left|\epsilon_{j}^{(1)}(\tau)\right\rangle$ has been eliminated in such a way that

$$
\left\langle\epsilon_{j}^{(1)}(\tau)\right|\left(\frac{d}{d \tau}\left|\epsilon_{j}^{(1)}(\tau)\right\rangle\right)=0
$$

Finally,

$$
\varphi_{j}^{(1)}(\tau)=\int_{0}^{\tau} d \tau^{\prime} \epsilon_{j}^{(1)}\left(\tau^{\prime}\right),
$$

where $\epsilon_{j}^{(1)}(\tau)$ is the perturbation value for $\epsilon_{j}(\tau)$, which, since

$$
\left\langle\epsilon_{j}^{(1)}(\tau)\left|(1 / T) K^{(1)}(\tau)\right| \epsilon_{j}^{(1)}(\tau)\right\rangle=0
$$

is precisely the eigenvalue of $H(\tau)[(3.7)]$.

In this approximation, the evolution operator is

$$
U^{(1)}(\tau)=S^{(1)}(\tau) \sum_{j} \exp \left[-i T \varphi_{j}^{(1)}(\tau) / \hbar\right]\left|\epsilon_{j}^{(1)}(0)\right\rangle\left\langle\epsilon_{j}^{(1)}(0)\right|
$$

and, as one would expect, coincides with the result which is obtained in the ordinary adiabatic theorem, ${ }^{1}$ which corresponds to an approximation of order $1 / T$.

\section{B. Second approximation}

We proceed now to calculate $\left|\epsilon_{j}^{(2)}(\tau)\right\rangle, S^{(2)}(\tau), K^{(2)}(\tau)$, and $\epsilon_{j}^{(2)}(\tau)$. Straightforward application of ordinary perturbation theory to $(3.6)$ gives

$$
\begin{aligned}
\left|\epsilon_{j}^{(2)}(\tau)\right\rangle= & \left|\epsilon_{j}^{(1)}(\tau)\right\rangle+\sum_{i \neq j} \frac{\left\langle\epsilon_{i}^{(1)}(\tau)\left|-(1 / T) K^{(1)}(\tau)\right| \epsilon_{j}^{(1)}(\tau)\right\rangle}{\epsilon_{j}^{(1)}(\tau)-\epsilon_{i}^{(1)}(\tau)} \\
& \times\left|\epsilon_{i}^{(1)}(\tau)\right\rangle,
\end{aligned}
$$

which, with (3.9), is

$$
\left|\epsilon_{j}^{(2)}(\tau)\right\rangle=\left|\epsilon_{j}^{(1)}(\tau)\right\rangle-(i / T) \sum_{i \neq j} c_{i j}(\tau)\left|\epsilon_{i}^{(1)}(\tau)\right\rangle,
$$

where

$$
\begin{aligned}
& c_{i j}^{(1)}(\tau)=\alpha_{i j}^{(1)}(\tau) / \omega_{j i}^{(1)}(\tau), \quad \alpha_{i j}^{(1)}(\tau)=\left\langle\epsilon_{i}^{(1)}(\tau)\right|\left(\frac{d}{d \tau}\left|\epsilon_{j}^{(1)}(\tau)\right\rangle\right), \\
& \omega_{i j}^{(1)}(\tau)=\frac{\epsilon_{i}^{(1)}(\tau)-\epsilon_{j}^{(1)}(\tau)}{\hbar} .
\end{aligned}
$$

In this order, $S^{(2)}(\tau)$ is given by

$$
S^{(2) \dagger}(\tau)\left|\epsilon_{j}^{(2)}(\tau)\right\rangle=\left|\epsilon_{j}^{(2)}(0)\right\rangle
$$

and $K^{(2)}(\tau)$ is

$$
\begin{aligned}
K^{(2)}(\tau)= & i \hbar \sum_{j}\left(\frac{d}{d \tau}\left|\epsilon_{j}^{(2)}(\tau)\right\rangle\right)\left\langle\epsilon_{j}^{(2)}(\tau)\right| \\
\approx & K^{(1)}(\tau)+\frac{\hbar}{T} \sum_{j} \sum_{i \neq j}\left\{\left(\frac{d}{d \tau} c_{i j}^{(1)}(\tau)\right)\left|\epsilon_{i}^{(1)}(\tau)\right\rangle\left\langle\epsilon_{j}^{(1)}(\tau)\right|\right. \\
& -c_{i j}^{(1) *}(\tau)\left(\frac{d}{d \tau}\left|\epsilon_{j}^{(1)}(\tau)\right\rangle\right)\left\langle\epsilon_{i}^{(1)}(\tau)\right| \\
& \left.+c_{i j}^{(1)}(\tau)\left(\frac{d}{d \tau}\left|\epsilon_{i}^{(1)}(\tau)\right\rangle\right)\left\langle\epsilon_{j}^{(1)}(\tau)\right|\right\} \\
\equiv & K^{(1)}(\tau)+\frac{\hbar}{T} \sum_{j} \sum_{i \neq j} F_{i j}^{(1)}(\tau) .
\end{aligned}
$$


Putting in (3.6) $(1 / T) K^{(2)}$ instead of $(1 / T) K$ and applying perturbation theory, one finds that to order $1 / T^{2}$ the contributions to $\epsilon_{j}^{(2)}(\tau)$ will come from the diagonal and nondiagonal terms of $K^{(1)}(\tau)$ and from the diagonal elements of $F_{i j}^{(1)}(\tau)$. Of all these, only the nondiagonal elements of $K^{(1)}$ contribute, giving the final result

$$
\epsilon_{j}^{(2)}(\tau)=\epsilon_{j}^{(1)}(\tau)+\frac{\hbar}{T^{2}} \sum_{i \neq j} \frac{\left|\alpha_{i j}(\tau)\right|^{2}}{\omega_{j 1}(\tau)} .
$$

The expression for the evolution operator in this approximation is thus

$$
\begin{aligned}
U^{(2)}(\tau)= & S^{(2)}(\tau) \\
& \times \sum_{j} \exp \left[-i T \int_{0}^{\tau}\left(\epsilon_{j}^{(1)}\left(\tau^{\prime}\right)+\frac{\hbar}{T^{2}} \sum_{i \neq j} \frac{\left|\alpha_{i j}\left(\tau^{\prime}\right)\right|^{2}}{\omega_{j i}}\right) d \tau^{\prime}\right] \\
& \times\left|\epsilon_{j}^{(2)}(0)\right\rangle\left\langle\epsilon_{j}^{(2)}(0)\right|,
\end{aligned}
$$

where everything has been previously calculated.

\section{C. $n$th approximation}

For $n$th order, $\left|\epsilon_{j}^{(n)}\right\rangle$ is obtained from $\left|\epsilon_{j}^{(n-1)}\right\rangle$ by taking $(1 / T) K^{(n-1)}(\tau)$ as the perturbation and calculating to order $1 / T^{n} . S^{(n)}$ is then determined by the relation

$$
S^{(n)}\left|\epsilon_{j}^{(n)}(0)\right\rangle=\left|\epsilon_{j}^{(n)}(\tau)\right\rangle
$$

and $K^{(n)}(\tau)$ given by

$$
K^{(n)}(\tau)=i \hbar \sum_{j}\left(\frac{d}{d \tau}\left|\epsilon_{j}^{(n)}(\tau)\right\rangle\right)\left\langle\epsilon_{j}^{(n)}(\tau)\right| .
$$

This allows one to calculate $\epsilon_{j}^{(n)}$ through the perturbing term - $(1 / T) K^{(n)}(\tau)$, and finally one would obtain

$$
\begin{aligned}
U^{(n)}(\tau)= & S^{(n)}(\tau) \sum_{j} \exp \left[-i T \int_{0}^{\tau} \epsilon_{j}^{(n)}\left(\tau^{\prime}\right) d \tau^{\prime}\right] \\
& \times\left|\epsilon_{j}^{(n)}(0)\right\rangle\left\langle\epsilon_{j}^{(n)}(0)\right|,
\end{aligned}
$$

which is the explicit expression for the evolution operator to order $(1 / T)^{n}$.

\section{AN EXAMPLE}

As an application of the described method we calculate now, in second order, the evolution operator of a system consisting of an atom in a magnetic field whose direction is reversed adiabatically. ${ }^{1}$

The Hamiltonian is given by

$$
H(t)=H^{(0)}+A(\mathrm{~L} \cdot \mathrm{S})-\frac{e}{2 \mu c}\left(L_{z}+2 S_{z}\right) H(t),
$$

where the magnetic field $H$ is always parallel to the $z$ axis and changes adiabatically from $H_{0}$ to $-H_{0}$ according to the law

$$
H(t)=H_{0}(2 \tau-1),
$$

$\tau$ being $t-t_{0} / T, T$ large.

Let us suppose that the system is initially in a state ${ }^{2} P$ with $J_{z}=\frac{1}{2}$. There are two eigenvectors of $H(t)$ corresponding to this value that will be linear combinations of the eigenstates $\left(\left|L_{z}, S_{z}\right\rangle\right)\left|0, \frac{1}{2}\right\rangle$ and $\left|1,-\frac{1}{2}\right\rangle$ of $H^{(0)}$. Taking the corresponding $H^{(0)}$ eigenvalue as the zero of energy, we get, in this basis, where

$$
\begin{aligned}
& \rho(\tau) \equiv \frac{e \hbar}{2 \mu c} \frac{H(\tau)}{A \hbar^{2}}, \quad \mathbf{u} \equiv\left(\frac{2 \sqrt{2}}{b}, 0, \frac{1-2 \rho}{b}\right), \\
& b \equiv\left[8+(1-2 \rho)^{2}\right]^{1 / 2}, \quad|\mathbf{u}|=1,
\end{aligned}
$$

and the $\sigma$ are the Pauli matrices.

The starting point of our procedure is the eigenvalue problem for $H(\tau)$ given by (4.3). The two solutions, labelled \pm , are

$$
\begin{aligned}
& \epsilon_{ \pm}^{(1)}(\tau)=\frac{1}{4} A \hbar^{2}[-1-2 \rho(\tau) \pm b(\tau)], \\
& \left|\epsilon_{+}^{(1)}(\tau)\right\rangle=\frac{1}{\sqrt{2\left(1+u_{z}\right)}}\left(\begin{array}{c}
1+u_{z} \\
u_{x}+i u_{y}
\end{array}\right)=\frac{1}{\sqrt{2 b(1-2 \rho+b)}}\left(\begin{array}{c}
1-2 \rho+b \\
2 \sqrt{2}
\end{array}\right) \\
& \left|\epsilon_{-}^{(1)}(\tau)\right\rangle=\frac{1}{\sqrt{2\left(1+u_{z}\right)}}\left(\begin{array}{c}
-u_{x}+i u_{y} \\
1+u_{z}
\end{array}\right)=\frac{1}{\sqrt{2 b(1-2 \rho+b)}}\left(\begin{array}{c}
-2 \sqrt{2} \\
1-2 \rho+b
\end{array}\right) .
\end{aligned}
$$

Now, $S^{(1)}(\tau)$ is determined by $(3.8)$, which in this case is

$$
S^{(1)}(\tau)\left|\epsilon_{ \pm}^{(1)}(0)\right\rangle=\left|\epsilon_{ \pm}^{(1)}(\tau)\right\rangle
$$

and gives

$$
\begin{aligned}
S^{(1)}(\tau)= & \frac{1}{\sqrt{3 b(1-2 \rho+b)}} \\
& \times\left(\begin{array}{cc}
3+b-2 \rho & -(1 / \sqrt{2})(3-b+2 \rho) \\
(1 / \sqrt{2})(3-b+2 \rho) & 3+b-2 \rho
\end{array}\right) .
\end{aligned}
$$

The evolution operator is now determined by (3.13), a result which corresponds to the ordinary adiabatic theorem, valid in order $1 / T$.

To obtain $U$ in second order, we need $\alpha_{i j}^{(1)}$ and $\omega_{i j}^{(1)}$. From (3.16), (4.5), and (4.6) we get

$$
\begin{aligned}
& \alpha_{+-}^{(1)}=-2 \sqrt{2} \frac{[(d / d \tau) \rho]}{b^{2}}=-\alpha_{-+}^{(1)}, \quad \alpha_{++}^{(1)}=\alpha_{--}^{(1)}=0, \\
& \omega_{+-}^{(1)}=\frac{1}{2} A \hbar b=-\omega_{-+}^{(1)},
\end{aligned}
$$

from which

$$
C_{+-}^{(1)}=\frac{-4 \sqrt{2}[(d / d \tau) \rho]}{b^{3} A \hbar}=C_{-+}^{(1)} \equiv C .
$$

With (4.11), Eq. (3.15) gives

$$
\begin{aligned}
\left|\epsilon_{+}^{(2)}(\tau)\right\rangle & =\frac{1}{\sqrt{2 b(1-2 \rho+b)}}\left(\begin{array}{c}
(1-2 \rho+b)+(i / T) 2 \sqrt{2} c \\
2 \sqrt{2}-(i / T)(1-2 \rho+b) c
\end{array}\right) \\
& \equiv\left(\begin{array}{l}
a_{1}(\tau) \\
a_{2}(\tau)
\end{array}\right), \\
\left|\epsilon_{-}^{(2)}(\tau)\right\rangle & =\frac{1}{\sqrt{2 b(1-2 \rho+b)}}\left(\begin{array}{c}
-2 \sqrt{2}-(i / T)(1-2 \rho+b) c \\
(1-2 \rho+b)-(i / T) 2 \sqrt{2} c
\end{array}\right) \\
& \equiv\left(\begin{array}{l}
b_{1}(\tau) \\
b_{2}(\tau)
\end{array}\right) .
\end{aligned}
$$

Finally, (3.17) defines $S^{(2)}(\tau)$, which in terms of the variables introduced in (4.12) is given by

$$
\begin{aligned}
S^{(2)}(\tau)= & \frac{1}{a_{1}(0) b_{2}(0)-a_{2}(0) b_{1}(0)} \\
& \times\left(\begin{array}{ll}
a_{1}(\tau) b_{2}(0)-a_{2}(0) b_{1}(\tau) & a_{1}(0) b_{1}(\tau)-a_{1}(\tau) b_{1}(0) \\
a_{2}(\tau) b_{2}(0)-a_{2}(0) b_{2}(\tau) & a_{1}(0) b_{2}(\tau)-a_{2}(\tau) b_{1}(0)
\end{array}\right),
\end{aligned}
$$


and the eigenvalues $\epsilon_{t}^{(2)}(\tau)$ are obtained from (3.19), (4. 9), and (4.10) with the result

$$
\epsilon_{ \pm}^{(2)}(\tau)=\frac{A \hbar^{2}}{4}(-1-2 \rho \pm b) \pm \frac{\hbar}{T^{2}} \frac{16[(d / d \tau) \rho]}{A \hbar b^{3}}
$$

Equations (4.13) and (4.14) determine completely the evolution operator $U^{(2)}$ by (3.20),

$$
\begin{aligned}
U^{(2)}(\tau)= & S^{(2)}(\tau)\left\{\exp \left[-i T \varphi_{+}^{(2)}(\tau) / \hbar\right]\left|\epsilon_{+}^{(2)}(0)\right\rangle\left\langle\epsilon_{+}^{(2)}(0)\right|\right. \\
& \left.+\exp \left[-i T \varphi_{-}^{(2)}(\tau) / \hbar\right]\left|\epsilon_{-}^{(2)}(0)\right\rangle\left\langle\epsilon_{-}^{(2)}(0)\right|\right\} \\
\varphi_{ \pm}^{(2)}(\tau)= & \int_{0}^{\tau} d \tau^{\prime} \epsilon_{ \pm}^{(2)}\left(\tau^{\prime}\right)
\end{aligned}
$$

The procedure can be continued to higher orders with increasing computational difficulties.

\section{CONCLUSIONS}

As stated previously, the advantage of the spinning axis representation proposed here lies in the fact that it allows one to calculate the evolution operator in the approximation $1 / T^{n}$ performing only one transformation $S^{(n)}$ on the original system. In the second approximation only $S^{(2)}$ is necessary; it is simple to see that the combined action of the $R^{(2)}, R^{(1)}$ of Ref. 4 is equivalent to the action of $S^{(2)}$, etc.
It should be remarked that although in the previous example we have calculated the explicit form of $S^{(1)}$ for the sake of completeness, it is not necessary to know it to obtain $S^{(2)}$ and that, in general, $S^{(n)}$ can be obtained directly without going through the succesive changes of representation of Ref. 4. This is the advantage of the method, since to calculate $S^{(n)}$ it is only necessary to apply straightforward perturbation theory, the perturbation being "finer" at each succesive step.

We finally mention that, taking into account that the role of the SAR is played in classical mechanics by a canonical transformation, the method of this paper could be extended to classical systems in a form similar to the one used in a previous work. ${ }^{5}$

${ }^{1}$ A. Messiah, Mécanique quantique (Dunod, Paris, 1964), Vol. II, Chap. XVII.

${ }^{2}$ K. O. Friedrichs, Reports IMM-NYU 281, New York University (1955). IMM-NYU 230, New York University (1956).

${ }^{3}$ T. Kato, J. Phys. Soc. Jap. 5, 435 (1950).

${ }^{4}$ L. Garrido, J. Math. Phys. 5, 355 (1964).

${ }^{5}$ L. Navarro and L, Garrido, J. Phys. A (Proc. Phys. Soc.) 1, 326 (1968). 